\title{
Effects of multidomain versus single- domain training on executive control and memory in older adults: study protocol for a randomized controlled trial
}

Soledad Ballesteros ${ }^{1,2^{*}}$, Jennifer A. Rieker ${ }^{1,2}$, Julia Mayas ${ }^{1,2}$, Antonio Prieto ${ }^{1,2}$, Pilar Toril ${ }^{1}$, María Pilar Jiménez $z^{1,2}$ and José Manuel Reales ${ }^{1,3}$

\begin{abstract}
Background: Previous research suggests that both cognitive training and physical exercise help to maintain brain health and cognitive functions that decline with age. Some studies indicate that combined interventions may produce larger effects than each intervention alone. The aim of this study is to investigate the effects of combined cognitive and physical training compared to cognitive training and physical training alone on executive control and memory functions in healthy older adults.

Objectives: The main objectives of this four-arm randomized controlled trial (RCT) are: to investigate the synergetic effects of a simultaneous, group-based multidomain training program that combines cognitive video-game training with physical exercise, in comparison to those produced by cognitive training combined with physical control activity, physical training combined with cognitive control activity, or a combination of both control activities; to investigate whether event-related potential latencies of the P2 component are shorter and N2 and P3b components assessed in a memory-based task switching task are enhanced after training; and to find out whether possible enhancements persist after a 3-month period without training.
\end{abstract}

Methods: In this randomized, single-blind, controlled trial, 144 participants will be randomly assigned to one of the four combinations of cognitive training and physical exercise. The cognitive component will be either video-game training (cognitive intervention, Cl) or video games not specifically designed to train cognition (cognitive control, CC). The physical exercise component will either emphasize endurance, strength, and music-movement coordination (exercise intervention, El) or stretching, toning, and relaxation (exercise control, EC).

Discussion: This RCT will investigate the short and long-term effects of multidomain training, compared to cognitive training and physical training alone, on executive control and memory functions in healthy older adults, in comparison with the performance of an active control group.

(Continued on next page)

\footnotetext{
* Correspondence: mballesteros@psi.uned.es

'Studies on Aging and Neurodegenerative Diseases Research Group, Universidad Nacional de Educación a Distancia, Madrid, Spain

2Department of Basic Psychology II, Facultad de Psicología, Universidad Nacional de Educación a Distancia, Juan del Rosal, 10, Madrid, Spain

Full list of author information is available at the end of the article
}

(c) The Author(s). 2020 Open Access This article is licensed under a Creative Commons Attribution 4.0 International License, which permits use, sharing, adaptation, distribution and reproduction in any medium or format, as long as you give appropriate credit to the original author(s) and the source, provide a link to the Creative Commons licence, and indicate if changes were made. The images or other third party material in this article are included in the article's Creative Commons licence, unless indicated otherwise in a credit line to the material. If material is not included in the article's Creative Commons licence and your intended use is not permitted by statutory regulation or exceeds the permitted use, you will need to obtain permission directly from the copyright holder. To view a copy of this licence, visit http://creativecommons.org/licenses/by/4.0/ The Creative Commons Public Domain Dedication waiver (http://creativecommons.org/publicdomain/zero/1.0/) applies to the data made available in this article, unless otherwise stated in a credit line to the data. 
(Continued from previous page)

Trial registration: ClinicalTrials.gov, NCT03823183. Registered on 21 January 2019.

Keywords: Aging, Cognitive training, Physical exercise, Multidomain training, Executive functions, Memory functions, Randomized controlled trial

\section{Background}

Age-related cognitive decline affects negatively the performance of daily living activities and the quality of life of many older adults. Neurocognitive frailty is the principal threat to successful aging $[1,2]$ as cognitive performance is central for daily life [3]. Cross-sectional studies have reported declines in a series of cognitive abilities [4-7], although these declines are less pronounced in longitudinal studies [5]. Aging is associated with a progressive decline in a wide range of cognitive abilities, such as set shifting [6], working memory [4, 6], and episodic memory $[5,7,8]$. Yet other cognitive functions which rely on previous experience, such as vocabulary and general knowledge $[4,9,10]$, procedural knowledge [11], and implicit memory [12-15], are mainly preserved, not only in healthy older adults but also in those with mild cognitive impairment [16], people with Alzheimer disease $[14,17]$, and older adults with type 2 diabetes mellitus [18].

Cerebral aging is associated with gray and white matter reduction in several areas of the brain, including the lateral prefrontal cortex, the cerebellum, and the medial temporal lobe system including the hippocampus. In contrast, minimal decreases occur in the entorhinal and occipital cortices [19]. The prefrontal cortex organizes the incoming information and interacts with the hippocampus while performing working-memory tasks [20, 21]. Cognitive-control functions refer to the ability to adapt behavior in order to process only relevant over competing irrelevant information to attain certain goals. Neuroanatomical changes occurring in the lateral prefrontal cortex and the medial temporal lobe-hippocampus complex are associated with declines in executive functions, working memory, and episodic memory. The failure of these basic cognitive functions predicts upcoming difficulties with the performance of daily-living activities and compromises independent living [22]. However, even in advanced age, the human brain preserves a certain degree of plasticity and functional reorganization, which allows people to adapt to agerelated cerebral changes in order to maintain successful task performance [23-25]. Neuroplasticity in older adults is contingent on individual behavior [26-30] and is susceptible to be modified by interventions designed to delay or prevent age-related cognitive decline [31]. Brain plasticity and its role in neural adaptations to age-related cerebral changes are also influenced by comorbidities, environmental factors, personality traits (psychosocial variables), and genetic and epigenetic factors [32]. A recent Frontiers Research Topic monograph focused on research conducted in the field of cognitive and brain plasticity induced by physical activity, cognitive training (computerized interventions, learning therapy, video games), and combined-intervention approaches, as well as other forms of brain stimulation that target brain activity, such as electroencephalography and neurofeedback [33]. During the last two decades, researchers have conducted a variety of intervention studies directed to promote behavioral flexibility and to enhance several cognitive processes that decline with age. Indeed, evidence for the benefits of cognitive training, video games, and physical exercise is growing rapidly, as well as research directed at gaining a better understanding of the underlying mechanisms and their translation to clinical practice [34-36].

Cognitive training is an intervention that allows structured training in a series of tasks relevant to different cognitive functions, such as executive functions, speed of processing, episodic memory, cognitive control, or attention. Among cognitive psychologists and neuroscientists there is increasing interest in exploring whether cognitive training with specially designed computerized training programs and video games of different kinds enhances cognition. Video games are electronic games that require interaction with a computer or other electronic devices with a user interface that provides visual and auditory feedback. Computerized cognitive programs and video games are currently receiving great attention in exploring the possibility of transfer to untrained tasks [37-42]. Many intervention studies based on cognitive training support the idea that training in older adults improves some aspects of cognition but not others. In recent years, several meta-analyses [43-47] have examined the effectiveness of computer-based interventions in healthy older adults. These meta-analytic studies have shown low to moderate training effects in older adults in several cognitive processes that decline with age, such as processing speed, attention, and memory. However, others [48] have reported that 
playing video games had little consequences on cognition. Due to different study designs (e.g., the inclusion of active or passive control groups $[49,50])$ and types of training (e.g., video games of different kinds, computerized cognitive programs [51-54]), results have been heterogeneous, making it difficult to reach solid conclusions [55].

In addition, other types of training such as physical activity of different kinds are also explored as a way to improve the physical and cognitive status. The term "physical activity" includes a large number of activities related to voluntary body movements [32]. A large body of evidence supports the beneficial effects of physical activity on executive functions and memory [56-61]. Although early physical activity intervention studies, which mainly centered on cardiovascular training, showed that cardiovascular activity produced increases in hippocampal volume in older adults while improving spatial memory performance [62, 63], other types of physical exercise, such as motor fitness and coordination training, also resulted in increased hippocampal volume in healthy older adults [60]. Complex physical activities such as dancing [64-66] or the practice of martial arts [67-71] have also shown beneficial effects on cognition in older adults.

Several studies suggest that social engagement plays a key role in the maintenance of cognitive functioning and psychological well-being in older adults [32, 72, 73] (for a recent review, see [74]). In the present multidomain intervention, social engagement is not considered a source of variance, as it is not a factor manipulated in the intervention, but, rather, a design feature included to enhance cognitive and physical functioning. So, cognitive and physical training, as well as their control activities, will be performed in a social environment. In this way, the four groups will be trained in the same social conditions; that is, in small groups and in the presence of a trainer.

\section{Objectives and hypotheses}

The main objective of this randomized controlled trial (RCT) is to investigate the synergetic effects of a groupbased multidomain training program that combines cognitive video-game training with physical exercise, in comparison to those produced by cognitive training combined with physical control activity, physical training combined with cognitive control activity, or a combination of both control activities, on behavioral and electrophysiological measures of executive control (setshifting, response inhibition, and information updating and monitoring) and memory functions (immediate and delayed visual and verbal memory). These cognitive functions, which are often compromised in later years, are essential for everyday activities. The second objective is to investigate whether event-related potential (ERP) latencies of the P2 component are shorter and N2 and P3b components assessed in a memory-based taskswitching task are enhanced after training. Electrophysiology provides a very useful online measure to identify the contribution of different processing stages of executive functioning. ERPs can help us to understand the specific executive control impairments occurring with age, as well as the possible effects of the different types of intervention investigated in this RCT. To this end, the task-switching paradigm is a valid task that helps to identify the cognitive processes that most decline with ageing [75]. However, electrophysiological studies conducted to evaluate training-related effects in older adults using this task are scarce [76]. Finally, we are interested in finding out whether possible enhancements persist after a 3-month period without training.

We expect to find greater behavioral improvements in executive control and memory functions after training, larger maintenance effects, and shorter ERP latencies of the P2 component and enhanced N2 and P3b components in the multidomain training condition in comparison to both single-domain conditions. We also expect the multidomain group and both single-domain groups to outperform the active control group at the 3-month follow-up period.

In this RCT, we will use questionnaire data to verify that the groups do not differ in their levels of intrinsic motivation and engagement. At the end of the assessment session, participants will report their expectations regarding their performance in the assessment tasks using a 5-point Likert scale. Moreover, at the 1st, 8th, and 16th training session, the participants will respond to questions about motivation and engagement for each of the training video games. These factors will be examined by comparing the intervention arms to the active control condition. The engagement and motivation data will be used in secondary analyses as covariates in order to rule out these factors as sources of variation in the primary outcome variables.

\section{Methods}

The design is a four-arm, parallel RCT designed to investigate the effectiveness of combined cognitive and physical training versus cognitive and physical training alone but combined with a control activity, in comparison to an active control group, to promote cognitive and neurofunctional improvements in older adults. Fig. 1 shows the Consolidated Standards of Reporting Trials flow diagram corresponding to the present study.

\section{Study design}

Participants will complete one of the four combinations of cognitive training with video games and physical 


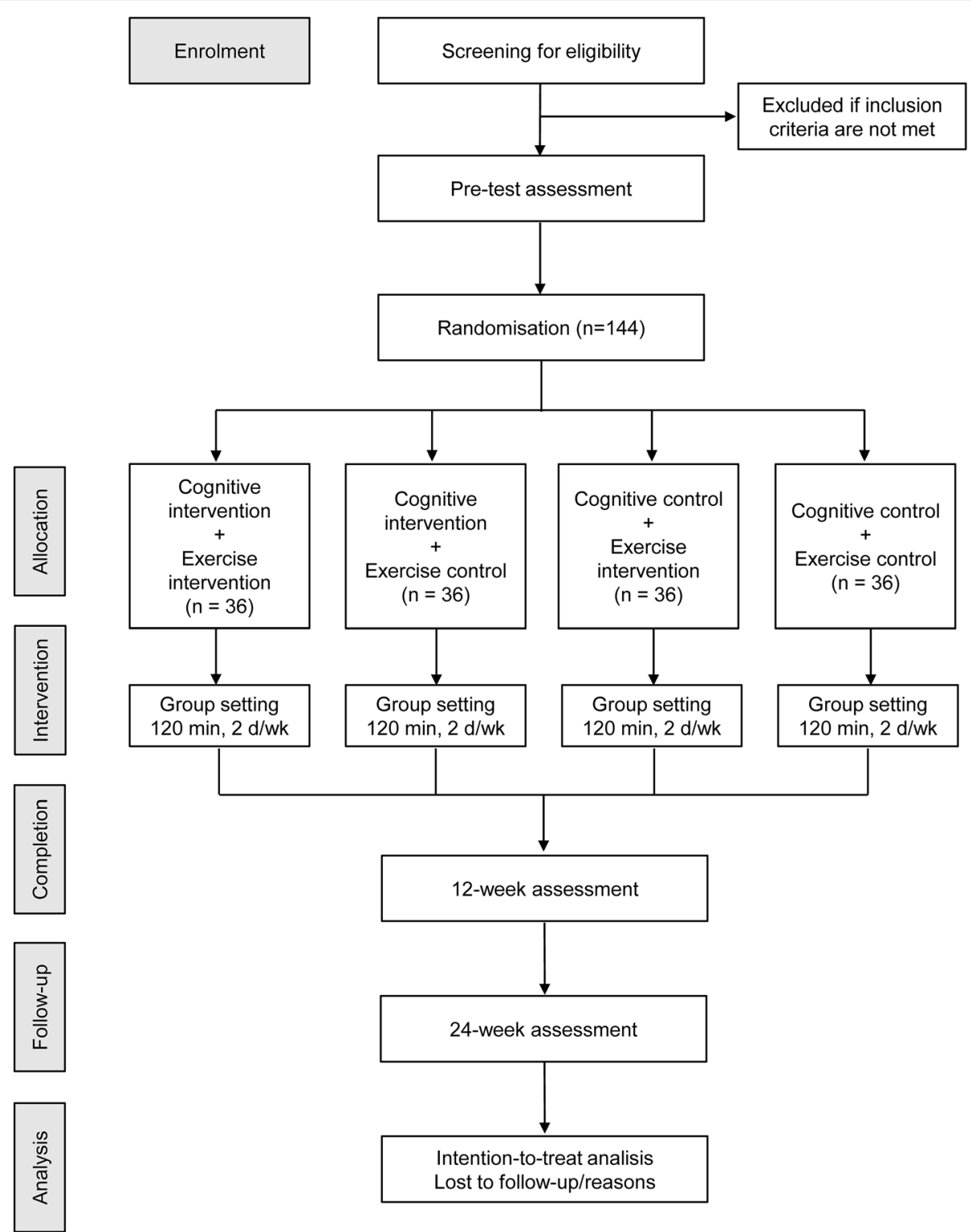

Fig. 1 Flow chart of the study protocol. d/wk days per week.

exercise. The cognitive component will be either a brain-training video-game program selected from Lumosity (cognitive intervention, $\mathrm{CI}$ ) or video games not specifically designed to train particular cognitive functions such as attention, memory, or executive control (cognitive control, CC). The physical exercise component will be either a senior-friendly adaption of BODYATTACK ${ }^{\mathrm{Tn}}$ (https://www.lesmills.com/), a combination of dance, aerobic, strength, and muscular resistance (exercise intervention, EI), or its control condition comprising stretching, toning, and relaxation (exercise control, EC). The duration of sessions for all groups will be the same, and all participants will perform physical exercise and video-gaming activities in a social environment (in small groups formed by 10-12 participants) and in the presence of the trainer.

To summarize, the study will have a $4 \times 3$ mixed factorial design with four intervention conditionsmultidomain $(\mathrm{CI}+\mathrm{EI})$, uni-domain cognitive intervention $(\mathrm{CI}+\mathrm{EC})$, uni-domain physical intervention (EI + $\mathrm{CC})$, and active control $(\mathrm{CC}+\mathrm{EC})$-assessed at three different time points (pretest, posttest, 3-month follow-up), with "Type of training" as between-subject factors and "Time" as the within-subject factor. The dependent variables will be behavioral and/or electrophysiological measures of executive functions (inhibition, shifting, working memory), memory functions (short term and long-term visual and word memory), 
and emotional well-being, quality of life, and motivation.

\section{Trial setting}

This study will be conducted in Madrid (Spain) at the UNED Psychology building. The screening, pretest, posttest, and follow-up assessments will be conducted in our laboratories at the Psychology building. The training sessions of the four groups will be conducted in three waves in spaces specifically equipped and prepared for this purpose at our university site as well as at a city council facility.

\section{Participants}

Participants will be male and female, healthy, and independently living volunteers aged between 60 and 80 years. Those who complete the baseline assessments and meet the inclusion criteria (see later) will be randomly assigned to one of the four intervention conditions. The timing of group allocation will take place between 1 and 8 weeks after baseline.

Participants may discontinue their intervention for personal or medical reasons. To minimize dropouts and improve adherence to the intervention, four face-to-face adherence reminder sessions will take place during the training program emphasizing the importance of training compliance. Furthermore, to increase participant retention and to reduce loss to follow-up, all participants will receive a personalized report of their performance and training progress at the end of the study. Participants will receive a small refund in compensation for their travelling expenses.

Even though this trial is low risk, participants might harm themselves during the practice of physical exercise. To minimize the risk of injuries, each participant will be carefully monitored during the training sessions. The interventions will be designed in collaboration with the exercise instructors, after a detailed analysis and taking into account each participant's possible medical issues. Spontaneously reported adverse events or other unintended effects will be registered and analyzed, and if necessary the protocol will be modified to eliminate the causing element. We have signed an insurance policy in case any participant suffers harm during the physical training.

\section{Inclusion and exclusion criteria}

Participants will have normal or corrected to normal vision and hearing, and will be free of neurological or musculoskeletal conditions, psychiatric conditions, or traumatic brain damage. They will not practice intense sports or other forms of physical exercise and will not play video games of any sort for more than $1 \mathrm{~h}$ per week. To determine eligibility, participants will be screened individually. Exclusion criteria will be a score of below 26 on the Mini-Mental State Examination (MMSE) [77], a score of 6 or more on the Yesavage Geriatric Depression Scale [78] (Spanish adaptation by Martínez et al. [79]), less than 20/60 vision with or without correction based on self-report, inability to complete the training activities, inability to communicate in Spanish, current plans to move to another city, and significant heart or lung disease.

\section{Sample size}

We conducted an a priori power analysis using G*Power 3.1 [80] to calculate the appropriate sample size. Using an $\alpha$ value of 0.05 , power of 0.80 , and a medium effect size $(f=0.38)$ for video-game training [45] and physical training [81], and four groups within the $F$-test family, a total sample size of 124 is required. Considering a dropout rate of $12 \%$, a total of 144 participants would be sufficient to detect significant main effects. According to this calculation, the adequate number of participants in each group (multidomain training, video-game training, physical activity training, and active control) is 36. According to this, we will set a sample size of 36 participants per arm, which is adequate for the experimental design. According to Montgomery et al. [82], with this number of participants the design would be underpowered to detect an interaction effect, as it would need a fourfold increase in sample size. However, given that lower interaction effects would not be clinically relevant, we decided to maintain our initial sample size estimation.

In the elaboration of this protocol, we have followed the SPIRIT 2013 explanation and elaboration guidance for reporting protocols of clinical trials [83].

\section{Recruitment}

Participants will be recruited through organized information sessions about the project at senior programs at universities and through radio advertisements.

\section{Randomization and blinding}

After the baseline assessments, participants will be randomly allocated to one of the four training protocols in a stratified process using the online tool Random Lists (move this to the other linehttps://www.randomlists. $\mathrm{com} /$ ). JMR will generate the random sequence and will assign participants to interventions. At first, participants who came in couples will be randomly allocated as a unit to one of the four groups, and afterward the same procedure will be performed with the individual participants. This procedure aims to minimize dropouts due to separating couples in different groups. Participants and exercise instructors will be blinded to treatment allocation (single-blind). Data analysis will not be blinded, as it 
will be performed by the investigators who actively collaborate in the study. We do not envision any reason why participants should be unblinded, either during the trial or at the end of the study.

\section{Interventions}

Participants will complete 16 training sessions of sequentially combined physical and cognitive training, or the corresponding control activities. Participants will be trained in small groups on 2 days per week for $2 \mathrm{~h}$. The first $60 \mathrm{~min}$ of each session will be dedicated to the exercise intervention (EI) or the exercise control activity (EC), followed by $60 \mathrm{~min}$ of cognitive training with video games $(\mathrm{CI})$ or the cognitive control activity $(\mathrm{CC})$. Both $\mathrm{CI}$ and $\mathrm{CC}$ will be conducted on tablets (Brigmton BTPC 1018OC). EI and EC will be led by physical exercise instructors and accompanied by a music soundtrack.

\section{Cognitive intervention}

In each session, participants in the $\mathrm{CI}$ group will play 10 video games selected from the commercial Lumosity computerized training program (http://lumosity.com/). Lumosity provides a series of games targeting the improvement of several cognitive functions. Table 1 presents a short description of the games and their trained domains. These functions are sensitive to age-related cognitive decline and closely related to the ability to perform activities of daily living, such as driving. The participant will play the games in a predetermined sequence, for approximately 5-10 $\mathrm{min}$ for each game. Each participant in the CI group will have a Lumosity user account assigned. These games are adaptivemeaning that as performance improves, the difficulty increases, progressively adjusting to the participant's performance level.

\section{Physical intervention}

The exercise intervention will consist of BODYAT$\mathrm{TACK}^{\mathrm{Tx}}$, which is a registered trademark of moderate to high-intensity training that combines aerobic exercises with strength and balance exercises. During the exercise protocol, participants will train at $65-80 \%$ of their maximum heart rate. The training sessions are predetermined by the distributer and comprise standardized movements, exercises, and music soundtracks (see Table 2). Exercises include large plyometric movements and more controlled movements, and train equally upper and lower body muscles with dynamic movement coordination. The sequence of exercises is as follows: 10-min warm-up, 35-min main phase (with active recovery between intervals), and 10-min cool-down.

\section{Cognitive control activity}

The cognitive control component will exclusively involve language-specific processes and crystalized knowledge (see Table 3). These domains are preserved with age, and even though an implication of executive functioning cannot be ruled out, this is clearly not the main active component. The cognitive control games are available within the gaming service Google Play Games, which mimics cognitive training platforms. This will create the impression of receiving an intervention, thereby reducing expectation biases. Participants will play $10-15 \mathrm{~min}$ each game in a predetermined sequence.

\section{Physical control activity}

The physical control activity will consist of BODYBALANCE $^{\text {tu }}$ (https://www.lesmills.com/), which is a music-

Table 1 Short description of the video games for the cognitive intervention

\begin{tabular}{|c|c|c|}
\hline Game name & Trained function & Description \\
\hline $\begin{array}{l}\text { Train of } \\
\text { Thought }\end{array}$ & Divided attention & The player directs trains to their matching station \\
\hline Assist Ants & Divided attention & The player prevents collisions by placing obstacles in their paths \\
\hline $\begin{array}{l}\text { Trouble } \\
\text { Brewing }\end{array}$ & Divided attention & The player simultaneously serves orders to different customers \\
\hline Playing Koi & Divided attention & $\begin{array}{l}\text { The player keeps track of which fish has already been fed, in a square of randomly } \\
\text { appearing fishes }\end{array}$ \\
\hline Memory Serves & $\begin{array}{l}\text { Working memory and divided } \\
\text { attention }\end{array}$ & The player matches different pieces of luggage to their corresponding owners \\
\hline Disillusion & Flexibility & The game consists of matching tiles with different shapes, colors, or symbols \\
\hline Ebb and Flow & Flexibility & The player swipes in the direction to which the leaves are moving or pointing \\
\hline Master Piece & Spatial reasoning & The player reorientates a shape so that it fills a hollow section \\
\hline Speed Pack & Visualization & The player has to fit the last item into an already filled suitcase \\
\hline $\begin{array}{l}\text { Highway } \\
\text { Hazards }\end{array}$ & Information processing & The player dodges obstacles in a race through a virtual desert \\
\hline
\end{tabular}


Table 2 Description of the exercise intervention

\begin{tabular}{lll}
\hline Activity & Trained function & Description \\
\hline Adaptation of "BODYATTACK ${ }^{\mathrm{TM} "}$ & Cardiovascular fitness & Aerobic exercises \\
& Endurance & Strength moves \\
& Coordination & Movements to music soundtrack \\
& Balance & Stabilization exercises \\
\hline
\end{tabular}

guided exercise that combines Tai Chi, Yoga, and Pilates exercises. The sequence of exercises of each session is as follows: 10-min warm-up with Tai Chi exercises; 35-min main phase with Yoga and Pilates exercises with a focus on breathing, stretching, balance, and strengthening of abdominal muscles; and 10-min cool-down with meditation and relaxation. The physical activity intervention and the physical control activity are briefly described in Table 4.

\section{General procedure}

After baseline, participants who meet the inclusion criteria will be randomly assigned to one of the four groups. The active control group was introduced in the design to control for placebo effects [84]. The main question is whether the multidomain group will outperform the single-domain groups at posttest, and whether these groups will outperform the active control group in a series of cognitive-control and memory tasks (see below). We focused on these cognitive domains because they deteriorate with age and are critical for independent living.

All methodological designs of primary and secondary outcomes are constructed using the rules of counterbalance and stimulus rotation. Response keys will be counterbalanced across conditions. The computerized tasks have been programmed with E-Prime 2.0 (Psychological Software Tools Inc.). Continuous EEG activity will be recorded in our laboratories with thin electrodes from 40 scalp sites using NuAmps amplifiers while participants perform the task-switching task.

\section{Outcome measures}

Each group will be assessed at three time points. Possible improvement will be assessed at posttest (12 weeks) and follow-up (24 weeks) using baseline (week 0) outcomes as a reference point. A schematic diagram of the time schedule of data collection for all outcome measures is shown in Fig. 2 (see also Additional file 1: SPIRIT checklist).

To report the primary and secondary outcomes, we will follow the outcome definition proposed by Saldanha et al. [85] and Zarin et al. [86] that includes the domain, the specific measurement, the specific metric, the method of aggregation, and the time points that will be used for analysis.

\section{Primary outcomes: training effects on cognitive functions Set-shifting}

Memory-based task switching Executive functions will be assessed with a memory-based task-switching paradigm. In this task [76, 87], digits from 1 to 9 (excluding number 5) are presented in white on a black background on the computer screen. A cue indicating the relevant task is presented simultaneously with the digit below the fixation point. The cue "NUM" indicates a numerical task (smaller or greater than 5), "PAR" the parity task (odd vs. even), and "TAM" (a diminutive for Spanish "tamaño" (size) font) the font-size task (small vs. large). Each stimulus is presented in small $(7 \mathrm{~mm} \times 10 \mathrm{~mm})$ and large $(12 \mathrm{~mm} \times 18 \mathrm{~mm})$ size. Participants will perform three single and two mixed blocks. In the single blocks, they have to process digits according to the one-task rule (i.e., numerical, parity, or font-size task only). In the

Table 3 Short description of the video games for the cognitive control condition

\begin{tabular}{lll}
\hline Game name & Trained function & Description \\
\hline Hangman & Lexical access & The player guesses a word by suggesting letters within a certain number of guesses \\
Grammar & Lexical access & The player chooses the correct spelling of a word within three possibilities \\
Definitions & Semantics and lexical access & The player chooses the correct word according to a given definition \\
Word search & Lexical access & The player identifies words hidden in a grid filled with letters \\
Crossword & Semantics and lexical access & The player constructs words by solving clues \\
Synonyms and antonyms & Semantics and lexical access & The player produces a word with a similar or opposite meaning of a given word \\
Trivia quiz & Crystalized knowledge & The player answers to questions of general knowledge \\
\hline
\end{tabular}


Table 4 Description of the activities of the exercise control condition

\begin{tabular}{lll}
\hline Game name & Trained function & Description \\
\hline Adaption of "BODYBALANCE ${ }^{\text {TM" }}$ & Flexibility & Stretching \\
& Relaxation & Respiratory exercises \\
\hline
\end{tabular}

memory-based mixed blocks, participants have to switch between different tasks within the block. In the cue block they are instructed to switch the rule after every three trials in the following order "NUM-NUM-NUMPAR-PAR-PAR-TAM-TAM-TAM", while a cue is presented in every trial simultaneously with the digit. In the memory block, participants are instructed to switch

\begin{tabular}{|c|c|c|c|c|c|}
\hline & \multicolumn{5}{|c|}{ STUDY PERIOD } \\
\hline & \multirow{2}{*}{$\begin{array}{c}\text { Enrolment } \\
-t_{1}\end{array}$} & \multirow{2}{*}{$\begin{array}{c}\text { Allocation } \\
0\end{array}$} & \multicolumn{3}{|c|}{ Post-allocation } \\
\hline TIMEPOINT $^{\star *}$ & & & Baseline & $\begin{array}{c}12 \text { weeks (post- } \\
\text { test) }\end{array}$ & $\begin{array}{c}24 \text { weeks } \\
\text { (follow-up) }\end{array}$ \\
\hline \multicolumn{6}{|l|}{ ENROLMENT: } \\
\hline \multirow{2}{*}{$\begin{array}{l}\text { Eligibility screen } \\
\text { Informed consent }\end{array}$} & $x$ & & & & \\
\hline & $x$ & & & & \\
\hline Allocation & & $x$ & & & \\
\hline \multicolumn{6}{|l|}{ INTERVENTIONS: } \\
\hline \multicolumn{6}{|l|}{$C l+E I$} \\
\hline \multicolumn{6}{|l|}{$C l+E C$} \\
\hline \multicolumn{6}{|l|}{$C C+E I$} \\
\hline \multicolumn{6}{|l|}{$C C+E C$} \\
\hline \multicolumn{6}{|l|}{ ASSESSMENTS: } \\
\hline \multicolumn{6}{|l|}{ Primary outcomes: } \\
\hline \multirow[t]{2}{*}{$\begin{array}{r}E R P+\text { Memory based } \\
\text { task switching }\end{array}$} & & & $x$ & $x$ & $\mathrm{x}$ \\
\hline & & & $x$ & $x$ & $x$ \\
\hline$n$-Back task & & & $x$ & $x$ & $x$ \\
\hline Stroop task & & & $x$ & $x$ & $x$ \\
\hline \multirow{3}{*}{$\begin{array}{r}\text { WMS-III Faces } \\
\text { immediate + delayed } \\
\text { WMS-III Word-Pair } \\
\text { immediate + delayed }\end{array}$} & & & $x$ & $x$ & $x$ \\
\hline & & & $x$ & $x$ & $x$ \\
\hline & & & $x$ & $x$ & $x$ \\
\hline Secondary outcomes: & & & $x$ & $x$ & $x$ \\
\hline PANAS & & & $x$ & $x$ & $x$ \\
\hline \multirow{2}{*}{$S P P B$} & & & $x$ & $x$ & $x$ \\
\hline & & & $x$ & $\mathrm{x}$ & $x$ \\
\hline
\end{tabular}

Footnote: CC cognitive control, $\mathrm{Cl}$ cognitive intervention, EC exercise control,El exercise intervention, ERP event-related potential, LSI Life Satisfaction Index, 6MWT 6-Minute Walk Test, PANAS Positive and Negative Affect Schedule, SPPB Short Physical Performance Battery, TMT Trail Making Test, WMS-III Wechsler Memory Scale-Third Edition, 6MWT 6-Minute Walk Test.

Fig. 2 Standard Protocol Items: Recommendations for Interventional Trials (SPIRIT 2013) diagram illustrating the schedule of enrolment, post allocation, and close-out for all assessments. CC cognitive control, Cl cognitive intervention, EC exercise control, El exercise intervention, ERP event-related potential, LSI Life Satisfaction Index, 6MWT 6-Minute Walk Test, PANAS Positive and Negative Affect Schedule, SPPB Short Physical Performance Battery, TMT Trail Making Test, WMS-III Wechsler Memory Scale-Third Edition 
the rule after every three trials in the same order, while "XXX" instead of a cue is presented; that is, participants have to keep track of the trial sequence in their working memory. When three consecutive errors are made, or no response is given, cues are presented on three consecutive trials to help participants to find the track. Single blocks consist of 35 trials each, and two mixed blocks consisting of switch and no-switch trials: a cued block (90 trials) and a memory block (90 trials). The mixed blocks are equal with respect to the stimulus type, response type, and frequency of task switch (33.3\%). The stimulus-response mapping of the three tasks is overlapping; that is, responses according to "smaller than 5", "even", and "small size" are assigned to the left key and "larger than 5", "odd", and "large size" to the right key. The assignment will be counterbalanced across participants. The outcomes of interest are mean reaction times (RTs) between groups corresponding to correct trials at pretest, posttest, and follow-up time points. The specific metric will be the change from baseline.

\section{Processing speed and flexibility}

Trail Making Test (TMT) The TMT is a neuropsychological test of visual attention and task switching. The test comprises two parts (A and B). Each part consists of 25 circles distributed over a sheet of paper. In Part A, the circles are numbered $1-25$, and the participant draws lines to connect the numbers in ascending order. In Part $B$, the circles include both numbers (1-13) and letters $(\mathrm{A}-\mathrm{L})$; the task consists of connecting the circles in an ascending pattern, but with the added task of alternating between the numbers and letters (i.e., $1-\mathrm{A}-2-\mathrm{B}-$ $3-C$, etc.). The total times in seconds for Parts A and B represent the TMT-A and TMT-B direct scores. Scores of TMT-A account for perceptual speed, whereas the BA difference score is an indicator of task-switching abilities. The outcomes of interest are the mean time scores of the difference score, TMT-B minus TMT-A, to assess task switching between groups at pretest, posttest, and follow-up time points. The specific metric will be the change from baseline.

\section{Working memory}

The $N$-back task is a continuous performance task to assess maintenance and updating of information in working memory. This task has been used with older adults [88-90]. Participants are presented with a sequence of stimuli (consonant letters), and indicate whether the last stimulus matches the one presented " $n$ " trials back by pressing one of two keys (one for "yes" or another for "no"). We used a three level $N$-back task. In the 0-back condition, the letter $\mathrm{X}$ is the target. We include the 0 back condition as an index of perceptual-motor speed to control for the role of speed of processing in working memory performance. In the 1-back condition, participants have to remember the stimulus presented just before the current stimulus; in the 2-back level, they have to remember the stimulus presented two positions before. Each participant first performs a practice block of 17 trials at each level, followed by the experimental trials. Each level contains three blocks of 27 trials (81 trials per level), yielding a total of 243 trials. Each block of 27 trials consists of 17 "nontargets" ("no" response) and 10 "targets" ("yes" response). The outcomes of interest are the mean accuracy between groups as assessed by HitsFalse alarms. The specific metric will be the change from baseline (pretest) to posttest and follow-up.

\section{Inhibitory control}

The Stroop interference effect reflects the extra time needed to resolve the conflict generated by an automatically processed irrelevant dimension. The Stroop task assesses response inhibition. We use the computerized Color-Word version of the Stroop task [42] with two different conditions: in the congruent condition, color name words match with the ink color; while in the incongruent condition, color names are printed in an incompatible ink color. In both conditions, participants are instructed to name the color of the ink as soon as possible. Longer response latencies and higher error rates on incongruent trials (when the color of the letters conflicts with the word) compared to congruent trials (when color and word match) constitute the Stroop effect. The Stroop effect correlates negatively with the efficiency of inhibitory control. The Stroop task contains 18 practice trials and two experimental blocks of 126 trials each, with a proportion of incongruent trials of $66 \%$. Responses are assigned to the keys "v", "b" and " $n$ ", and the stimulus-response mapping is counterbalanced across participants. The dependent variable is the mean RT corresponding to the congruent and incongruent correct trials of the groups at pretest, posttest, and follow-up. The specific metric will be the change from pretest in the computerized version of the Stroop task to assess response inhibition.

\section{Immediate and differed visual and verbal memory}

Wechsler Memory Scale-Third Edition (WMS-III) Faces The WMS-III Faces subtest [91] (The Psychological Corporation, 1997) uses a recognition paradigm to assess immediate and delayed visual memory. In Faces I, participants are presented with 24 target faces at a speed of $2 \mathrm{~s}$ per picture. Then, they are shown 48 faces (24 targets and 24 distractors) and are asked to identify the target faces by responding either "yes" or "no" to each face. Participants are prompted to keep the target 
faces in mind. In Faces II, participants are shown 48 faces (24 targets and 24 distractors) after a 30-min delay and are asked to identify the target faces. The Hits-False alarms mean between groups at pretest, posttest, and follow-up assessments will be the outcome of interest. The specific metric will be the change from pretest to posttest and follow-up.

Wechsler Memory Scale-Third Edition (WMS-III) Word-Pair List The WMS-III Word-Pair subtest assesses immediate and delayed verbal memory. In this test, four trials of eight unrelated word pairs are presented at a rate of $3 \mathrm{~s}$ per pair. In the immediate recall condition, after the presentation of the four lists, the first word of each pair is read to the participant, who has to provide the associated word of the pair. After a delay of approximately $25-35 \mathrm{~min}$, the same procedure is repeated, and the participant provides the second word of each pair. Finally, a recognition task is administered where 24 word pairs are presented and the participant is asked to identify the pair as either "new" or "old". The Hits-False alarms mean between groups at pretest, posttest, and follow-up assessments will be the outcome of interest. The specific metric will be the change from pretest to posttest and follow-up.

\section{Electrophysiological measures}

Electroencephalograph acquisition While performing the experimental memory-based switching task, continuous electroencephalograph (EEG) activity will be recorded using a NuAmps amplifier (Neuroscan Inc.) inside a soundproof, electromagnetically shielded room. We will use a 34-channel elasticized Quik-Cap with Ag/ $\mathrm{AgCl}$ sintered electrodes (American Medical EEG Association, 1991). To control ocular artifacts, vertical and horizontal electrooculograms will be recorded in two bipolar channels. Eye blinks and vertical eye movements will be monitored via electrodes located below and on the supraorbital ridge of the left eye. Horizontal artifacts will be monitored via electrodes on the outer canthus of each eye. Linked mastoids (A1, A2) will be used as a reference, and participants will be grounded to the $\mathrm{AFz}$ electrode. All data will be digitized using a NuAmps amplifier in continuous recording mode. The sampling rate will be $1000 \mathrm{~Hz}$, and all channels will be online bandpass filtered $(0.1-140 \mathrm{~Hz})$ and notch filtered $(50 \mathrm{~Hz})$ to eliminate power line artifacts. Continuous data will be filtered offline using a digital Butterworth filter (0.1-40 $\mathrm{Hz} ; 12 \mathrm{~dB}$ per octave roll-off), an infinite impulse response filter that achieves a given filtering characteristic. After filtering, data will be separated into baselinecorrected and nonoverlapping epochs time-locked to the target onset. Epochs containing high amplitude/ frequency and muscle or other irregular artifacts will be removed by visual inspection. Only artifact-free epochs from correct trials will be selected for averaging. The existence of blinks and other ocular movements will not be a criterion for epoch rejection. This kind of artifact will be eliminated using Independent Component Analysis (ICA) [92-98]. After submitting EEG data to ICA decomposition, artifactual components will be removed by inspection of their activity, scalp topography, and spectral power. The length of the epoch in the targetlocked ERP will be $1100 \mathrm{~ms}$, and $600 \mathrm{~ms}$ in the responselocked ERP. We focus on P2, N2, and P2b. Analyses will be centered on the posttarget and postresponse ERPs at the midline electrodes located at the frontal, central, and parietal lobes $(\mathrm{Fz}, \mathrm{Cz}$, and $\mathrm{Pz})$ where the components of interest are usually maximum. P2 is a positive ERP component associated with retrieval of stimulus-response sets that will be measured between 150 and $300 \mathrm{~ms}$. N2 will be measured at the most negative pick between 150 and $400 \mathrm{~ms}$ after target onset. P3b will be measured in the time window of 300-600 ms after target onset. This wave is associated with context updating and working memory (see [75, 76, 87]).

\section{Secondary outcomes \\ Assessment of emotional and affective well-being}

The Positive and Negative Affect Schedule (PANAS) The PANAS [99] is a self-report questionnaire designed to assess the affective state. It consists of two 10-item scales to measure both positive and negative affect. Positive affect reflects the point to which a person feels enthusiastic, active, and alert, with energy and rewarding participation. Negative affect represents a general dimension of subjective distress and unpleasant participation that includes a variety of aversive states, such as disgust, anger, guilt, fear, and nervousness. Participants in the PANAS respond to a 20 -item test using a 5-point scale that ranges from very slightly or not at all (1) to extremely (5). We use the Spanish version [100] that provides good consistency and reliability indexes, and also confirms the original two factors of the questionnaire. The reliability (Cronbach's $\alpha$ ) and validity, both convergent and discriminant, have also been corroborated in the elderly Spanish population [101].

The outcomes of interest are the mean score per group of positive and negative affect assessed with the PANAS questionnaire at three time points: pretest, posttest, and follow-up. The specific metric will be the change from baseline.

The Life Satisfaction Index (LSI) The LSI [102] is a 20-item self-report questionnaire to measure psychological well-being in older adults. The instrument 
consists of five subscales, including zest for life (four items), resolution and fortitude (five items), congruence between desired and achieved goals (three items), positive self-concepts (three items), and mood tone. Respondents express their agreement or disagreement with the statements based on a 3-point Likert scale (agree $=2$ points; disagree $=1$ point; and "don't know" $=0$ points). The higher the overall score, the higher the individual's life satisfaction.

The outcome of interest is the mean score per group of the individual's life satisfaction assessed with the LSI questionnaire at three time points: pretest, posttest, and follow-up. The specific metric will be the change from baseline.

\section{Assessment of physical condition}

The Short Physical Performance Battery (SPPB) The SPPB [103] measures functional status and physical performance. First described in 1994, it is a composite measure assessing walking speed, standing balance, and sit-to-stand performance. The SPPB is calculated from three components: the ability to stand for up to $10 \mathrm{~s}$ with feet positioned in three ways (together side by side, semi-tandem, and tandem); time to complete a 3-m or 4-m walk; and time to rise from a chair five times.

Lower-extremity physical performance is assessed in the study with a composite measure of walking speed, standing balance, and sit-to-stand performance. The outcome will be the performance mean in the battery of the groups at three time points: pretest, posttest, and followup. The specific metric will be the mean change from pretest to the other time points.

The 6-Minute Walk Test (6MWT) The 6MWT [104] is commonly used to assess exercise capacity. The participant walks for $6 \mathrm{~min}$ as fast as possible. The primary outcome is the distance completed. The test is administered in accordance with the protocol endorsed by the ATS [105]. The test is performed on a straight 30-m corridor and all participants receive standardized scripted instructions and scripted phrases of encouragement each minute during the test. Besides the distance, monitored parameters are changes in oxygen saturation $\left(\mathrm{SpO}_{2}\right)$, and pretest and posttest dyspnea and fatigue using the Borg scale [106].

Functional capacity is assessed in the study with the 6MWT. The outcome measure will be the mean absolute value in the test obtained by the groups at three time points: pretest, posttest, and follow-up. The specific metric will be the mean change from pretest to the other time points of the study.

\section{Statistical analysis}

All data from participants with complete baseline assessment and who attended at least one training session will enter into a primary intention-to-treat analysis. For the secondary per-protocol analysis, only the data of participants with a complete cognitive assessment and an attendance rate $\geq 70 \%$ will be considered.

Executive functions (set shifting, maintenance, inhibitory control) and memory functions (short-term, visual, and verbal immediate and delayed memory) will be assessed at pretest, posttest, and follow-up. The statistical analysis corresponding to the behavioral results will be carried out with the SPSS statistical package for Windows (SPSS 25.0; IBM Corporation). Results will be considered significant at $p<0.05$, with Bonferroni-corrected post hoc tests performed as appropriate. We will explore the missing data to ascertain their pattern and will apply an adequate technique of multiple imputation. Repeated ANOVA measures will be conducted with four groups (multidomain training, cognitive training, exercise training, active control) at three time points (pretest, posttest, follow-up) to test the primary hypothesis (i.e., differences in efficacy between interventions compared to the active control condition). Repeated ANOVA measures will also be performed to determine the effect of the interventions on secondary outcomes. To evaluate the effect size of the combined multimodal group versus each individual intervention group and the control arm, we will use multimodal regression with an interaction term. Electrophysiological data will be analyzed with CURRY 8.0.2, the EEGLAB toolbox [92], and the ERPLAB plugin for EEGLAB [98].

\section{Data monitoring committee and data management}

Personal information about participants obtained during the individual interviews as well as performance data and all study-related information will be coded in a database and stored securely at the study site to maintain participant confidentiality. A coded identification (ID) number to maintain participant confidentiality will identify all data collection and administrative forms. The electronic data will be stored securely on a university computer and a high-drive disc (HDD) that are password protected. Paper copies as well as HDDs will be securely stored in a locked cabinet at the study site. All forms, lists, appointment records, consent forms, and any other listings that link participant ID numbers to other identification information will be stored in a separate, locked file in a limited access area. Only the members of the researcher team directly involved in data collection, maintenance, and management will have access to the data set.

The data monitoring committee (DMC) will be composed of JAR and JMR, who will regularly check on the 
correctness of data collection and encoding and its correspondence with the entrances in the laboratory diary. The DMC will be responsible for securing the data on a weekly basis on the aforementioned devices. The data will be stored securely in our laboratory for 5 years.

We have not planned to conduct subgroups of interim analyses.

\section{Steering committee}

The steering committee will meet at least on a quarterly basis to monitor the trial processes, independently of the funding organization. The committee will check compliance with the assessment and training protocols and the timelines, and will oversee and manage the trial. Its members, who form an active part of the research group, are SB and JMR. They will verify trial processes, such as participant enrollment, informed consent, eligibility, allocation of participants to groups, and adherence to trial interventions.

\section{Dissemination plans}

After completion of the trial, the results will be presented at international and national conferences and will be published in appropriate scientific journals. We will also deliver the results to the participants.

\section{Discussion}

We investigate the potential for cognitive training and physical exercise to prevent or minimize the negative effects occurring with aging. This clinical trial examines the efficacy of a combined intervention on moderate cognitive decline as well as affective well-being and physical condition in healthy older adults. This multimodal intervention study will contribute to the increasing body of literature investigating ways to promote brain plasticity and maintain healthy and active aging.

To summarize, cognitive decline and physical decline have negative effects in older adults and impact negatively on society due to the increasing number of older adults that will suffer cognitive decline and neurodegenerative diseases in the next decades. Finding effective ways to prevent the negative impact of declining cognition would have a key effect on the current limited social and health care resources.

\section{Trial status}

This clinical trial was registered at the National Institute of Health (NIH) with the Clinicaltrials.gov identifier NCT03823183 (https://register.clinicaltrials.gov/ClinicalTrials.gov) on 21 January 2019. The protocol version number is number 1 (January 2019). Recruitment started in February 2019 and is expected to be completed in February 2020. Once the trial is completed, results will be reported according to the Consolidated Standards of
Reporting Trials (CONSORT) guidelines. The trial is active and ongoing. We expect to have the final results by the middle of 2021.

\section{Supplementary information}

Supplementary information accompanies this paper at https://doi.org/10. 1186/s13063-020-04293-3.

Additional file 1. Standard Protocol Items: Recommendations for Interventional Trials (SPIRIT) checklist.

Additional file 2. Table of items found in the WHO trial registry data.

\section{Authors' contributions}

SB is principal investigator (PI) 1 and JMR is PI 2; both conceptualized the design with the help of the rest of the authors. SB and JAR wrote the article with the approval of the rest of the authors. JAR contributed to all the phases of the research, including enrollment of the participants, appointments, assessments, cognitive training, data analyses, and paper preparation. SB and JM prepared the protocol registration of the clinical trial. JMR provided statistical support and contributed to sample size calculation. AP contributed to the trial design and physical intervention. The other members of the group are also involved in the design, interpretation, and reporting of the trial. We do not intend to use professional writers. All of the individuals who have made significant contributions to the design, conduct, interpretation, and reporting of the results of this clinical trial will be recognized as authors on the final trial report. All authors have read and approved the final version of this manuscript.

\section{Funding}

The study is supported by a grant from the Spanish Ministry of Economy and Competitiveness (grant \# PSI2016-80377-R) to SB and JMR, and by a grant from the European Community to SB (grant \# H2020-SC1-DTH-03-2018, grant agreement $N^{\circ} 826506$, sustAGE). JAR is supported by a Doctoral fellowship (Spanish Ministry of Economy and Competitiveness: BES-2017-079760). AP is supported by a postdoctoral contract associated with the grant \#EC H2020-SCI-DTH-03-2018. The founders have no role in the study design, data collection and analysis, decision to publish, or preparation of the manuscript. None of the authors have any commercial/financial connections with Lumosity. The authors thank the participants for their collaboration.

\section{Availability of data and materials}

Access to the protocol and the dataset may be provided upon request to the authors.

\section{Ethics approval and consent to participate}

This clinical trial is registered on the ClinicalTrials.gov database (NCT03823183). The Institutional Review Board of the Universidad Nacional de Educación a Distancia (UNED) approved the study protocol. All participants will sign a written consent form at the beginning of the study and will be informed that they can cease participation at any time. A written information form in Spanish will be provided to the participant during the first visit to the laboratory. The form includes information about the objectives of the study and a description of the investigation, as well as potential benefits and risks. The form invites the participant to ask the investigator all of the questions she/he considers necessary. The participant will be informed that participation in the study is voluntary and they can withdraw at any time for any reason. The written form will inform the participant that all personal data will be treated with confidentiality, according to the norms applicable to this type of study. The principal investigator will sign the information form. After answering all of the questions formulated by the participant, she/he will read the informed consent form. This form will assure that the participant has read the information form, has understood all its terms, has been sufficiently informed, and has been able to ask questions about the objectives and methodology of the study in which she/he is going to participate. The consent form will inform the participants of their right to refuse participation or to withdraw consent at any time as participation is voluntary and they can stop their participation at any time without giving explanations and 
without any undesirable repercussion. The form will include the name of the participant and the date on which the participant signs the informed consent form.

The UNED Research Ethics Committee approved the study. The study will be conducted in accordance with the Declaration of Helsinki (World Medical Association, 2013)

\section{Competing interests}

None declared.

\section{Author details}

'Studies on Aging and Neurodegenerative Diseases Research Group, Universidad Nacional de Educación a Distancia, Madrid, Spain. ${ }^{2}$ Department of Basic Psychology II, Facultad de Psicología, Universidad Nacional de Educación a Distancia, Juan del Rosal, 10, Madrid, Spain. ${ }^{3}$ Department Methodology of Behavioral Sciences, Facultad de Psicología, Universidad Nacional de Educación a Distancia, Madrid, Spain.

\section{Received: 29 March 2019 Accepted: 30 March 2020}

Published online: 14 May 2020

\section{References}

1. Park D, Reuter-Lorenz P. The adaptive brain: aging and neurocognitive scaffolding. Annu Rev Psychol. 2009;60:173-96 https://doi.org/10.1146/ annurev.psych.59.103006.093656.

2. Reuter-Lorenz PA, Park DC. How does it STAC up? Revisiting the scaffolding theory of aging and cognition. Neuropsychol Rev. 2014;24:355-70 https:// doi.org/10.1007/s11065-014-9270-9.

3. Salthouse T. Consequences of age-related cognitive declines. Annual Rev Psychol. 2012;63:201-26 https://doi.org/10.1146/annurev-psych-120710100328

4. Park DC, Lautenschlager G, Hedden T, Davidson NS, Smith AD, Smith PK Models of visuospatial and verbal memory across the adult life span. Psychol Aging. 2002;17(2):299-320 https://doi.org/10.1037/0882-7974.17.2.299.

5. Rönnlund M, Nyberg L, Bäckman L, Nilsson LG. Stability, growth, and decline in adult life span development of declarative memory: cross-sectional and longitudinal data from a population-based study. Psychol Aging. 2005;20(1): 3-18 https://doi.org/10.1037/0882-7974.20.1.3.

6. Reimers S, Maylor EA. Task switching across the life span: effects of age on general and specific switch costs. Dev Psychol. 2005:41(4):661-71 https:// doi.org/10.1037/0012-1649.41.4.661.

7. Bopp KL, Verhaeghen P. Aging and verbal memory span: a meta-analysis. J Gerontol B Psychol Sci Soc Sci. 2005;60(5):223-33 https://doi.org/10.1093/ geronb/60.5.P223.

8. Nilsson L. Memory function in normal aging. Acta Neurol Scand Suppl. 2003;107:7-13 https://doi.org/10.1034/j.1600-0404.107.s179.5.x.

9. Verhaeghen P. Aging and vocabulary scores: a meta-analysis. Psychol Aging. 2003;18(2):332-9 https://doi.org/10.1037/0882-7974.18.2.332

10. Bialystok E, Craik F. Lifespan cognition: mechanisms of change. Oxford: Oxford University Press; 2006

11. Mireles DE, Charness N. Computational explorations of the influence of structured knowledge on age-related cognitive decline. Psychol Aging. 2002;17(2):245-59 https://doi.org/10.1037/0882-7974.17.2.245.

12. Fleischman DA, Gabrieli JD. Repetition priming in normal aging and Alzheimer's disease: a review of findings and theories. Psychol Aging. 1998; 13(1):88-119.

13. Mitchell DB, Bruss PJ. Age differences in implicit memory: conceptual, perceptual, or methodological? Psychol Aging. 2003;18(4):807-22 https:// doi.org/10.1037/0882-7974.18.4.807.

14. Ballesteros S, Reales JM. Intact haptic priming in normal aging and Alzheimer's disease: evidence for dissociable memory systems. Neuropsychologia. 2004:42(8):1063-70 https://doi.org/10.1016/j. neuropsychologia.2003.12.008.

15. Sebastián M, Ballesteros S. Effects of normal aging on event-related potentials and oscillatory brain activity during a haptic repetition priming task. Neuroimage. 2012;60(1):7-20 https://doi.org/10.1016/j.neuroimage. 2011.11.060

16. Ballesteros S, Mayas J, Reales JM. Cognitive function in healthy, aging and mild cognitive impaired older adults. Psicothema. 2004;25:18-24 https://doi. org/10.7334/psicothema2012.181.
17. Ballesteros S, Reales JM, Mayas J, Heller MA. Selective attention modulates visual and haptic repetition priming: effects on aging and Alzheimers' disease. Exp Brain Res. 2008;189(4):473-83 https://doi.org/10.1007/s00221008-1441-6.

18. Redondo MT, Beltrán-Brotóns $J$, Reales JM, Ballesteros S. Word-stem priming and recognition in type 2 diabetes mellitus, Alzheimer's disease and cognitively healthy older adults. Exp Brain Res. 2015;233:3163-74 https://doi.org/10.1007/s00221-015-4385-7.

19. Raz N, Lindenberger U, Rodrigue KM, Kennedy KM, Head D, Williamson A, et al. Regional brain changes in aging healthy adults: general trends, individual differences and modifiers. Cereb Cortex. 2005;15(11):1676-89 https://doi.org/10.1093/cercor/bhi044.

20. Baddeley A. Working memory: looking back and looking forward. Nat Rev Neurosci. 2003;4(10):829-39 https://doi.org/10.1038/nrn1201.

21. Dennis N, Kim H, Cabeza R. Age-related differences in brain activity during true and false memory retrieval. J Cogn Neurosci. 2008;20(8):1390-402 https://doi.org/10.1162/jocn.2008.20096.

22. Owsley C, Sloane M, McGwin G, Ball K. Timed instrumental activities of daily living tasks: relationship to cognitive function and everyday performance assessments in older adults. Gerontology. 2002;48(4):254-65 https://doi.org/ 10.1159/000058360

23. Ballesteros S, Bischof GN, Goh JO, Park DC. Neural correlates of conceptual object priming in young and older adults: an event-related fMRI study. Neurobiol Aging. 2013;34(4):1254-64 https://doi.org/10.1016/j. neurobiolaging.2012.09.019.

24. Osorio A, Pouthas V, Fay S, Ballesteros S. Ageing affects brain activity in highly educated older adults: an ERP study using a word-stem priming task. Cortex. 2010:46(4):522-34 https://doi.org/10.1016/j.cortex.2009.09.003.

25. Sebastián M, Reales JM, Ballesteros S. Aging affects event-related potentials and brain oscillations: a behavioral and electrophysiological study using a haptic recognition memory task with familiar objects. Neuropsychologia. 2011:49(14):3967-80 https://doi.org/10.1016/j.neuropsychologia.2011.10.013.

26. Pascual-Leone A, Amedi A, Fregni F, Merabet LB. The plastic human brain cortex. Annu Rev Neurosci. 2005;28:377-401 https://doi.org/10.1146/ annurev.neuro.27.070203.144216.

27. Li S, Brehmer $Y$, Shing $Y L$, Werkle-Bergner $M$, Lindenberger $U$. Neuromodulation of associative and organizational plasticity across the life span: empirical evidence and neurocomputational modeling. Neurosci Biobehav Rev. 2006;30(6):775-90 https://doi.org/10.1016/j.neubiorev.2006.06. 004.

28. Lövdén M, Bäckman L, Lindenberger U, Schaefer S, Schmiedek F. A theoretical framework for the study of adult cognitive plasticity. Psychol Bull. 2010;136(4):659-76 https://doi.org/10.1037/a0020080.

29. Brehmer $Y$, Kalpouzos G, Wenger E, Lövden M. Plasticity of brain and cognition in older adults. Psychol Res. 2014;78(6):790-802 https://doi.org/10. 1007/s00426-014-0587-z

30. Styliadis C, Kartsidis P, Paraskevopoulos E, loannides AA, Bamidis PD. Neuroplastic effects of combined computerized physical and cognitive training in elderly individuals at risk for dementia: an eLORETA controlled study on resting states. Neural Plast. 2015;2015:e172192 https://doi.org/10. 1155/2015/172192.

31. Ball K, Berch DB, Helmer KF, Jobe JB, Leveck MD, Marsiske M, et al. Effects of cognitive training interventions with older adults: a randomized controlled trial. JAMA. 2002;288(18):2271-81 https://doi.org/10.1001/jama.296.23.2805.

32. Ballesteros $\mathrm{S}$, Kraft $\mathrm{E}$, Santana $\mathrm{S}$, Tziraki $\mathrm{CH}$. Maintaining older brain functionality: a targeted review. Neurosci Biobehav Rev. 2015;55:453-77 https://doi.org/10.1016/j.neubiorev.2015.06.008.

33. Ballesteros S, Voelcker-Rehage C, Bherer L. Editorial: Cognitive and brain plasticity induced by physical exercise, cognitive training, video games, and combined interventions. Front Hum Neurosci. 2018;12:169 https://doi.org/ 10.3389/fnhum.2018.00169.

34. Raz N, Lindenberger U. Life-span plasticity of the brain and cognition: from questions to evidence and back. Neurosci Biobehav Rev. 2013;37:2195-200 https://doi.org/10.1016/j.neubiorev.2013.10.003.

35. Stanmore E, Stubbs B, Vancampfort D, de Bruin ED, Firth J. The effects of active video games on cognitive functioning in clinical and nonclinical populations: a meta-analysis of randomized controlled trials. Neurosci Biobehav Rev. 2017;78:34-43 https://doi.org/10.1016/j. neubiorev.2017.04.011

36. Zhu $\mathrm{X}$, Yin $\mathrm{S}$, Lang $\mathrm{M}$, He R, Li J. The more the better? A meta-analysis on effects of combined cognitive and physical intervention on cognition in 
healthy older adults. Ageing Res Rev. 2016;31:67-79 https:/doi.org/10.1016/ j.arr.2016.07.003.

37. Mozolic JL, Long AB, Morgan AR, Rawley-Payne M, Laurienti PJ. A cognitive training intervention improves modality-specific attention in a randomized controlled trial of healthy older adults. Neurobiol Aging. 2011;32(4):655-68 https://doi.org/10.1016/..neurobiolaging.2009.04.013.

38. Anguera JA, Boccanfuso J, Rintoul JL, Al-Hashimi O, Faraji F, Janowich J, et al. Video game training enhances cognitive control in older adults. Nature. 2013;501(7465):97-101 https://doi.org/10.1038/nature12486.

39. Basak C, Boot WR, Voss MW, Kramer AF. Can training in a real-time strategy video game attenuate cognitive decline in older adults? Psychol Aging. 2008:23(4):765-77 https://doi.org/10.1037/a0013494.

40. Ballesteros S, Prieto A, Mayas J, Toril P, Pita C, Ponce de León L, Reales JM, Waterworth J. Training older adults with non-action video games enhances cognitive functions that decline with aging: a randomized controlled trial. Front Aging Neurosci. 2014;6:277 https://doi.org/10.3389/fnagi.2014.00277.

41. Toril P, Reales JM, Mayas J, Ballesteros S. Brain training with video games enhances visuospatial working memory in older adults. Front Aging Neurosci. 2016;10:206 https://doi.org/10.3389/fnhum.2016.00206.

42. Ballesteros S, Mayas J, Prieto A, Ruíz-Márquez E, Toril P, Reales JM. Effects of video game training on measures of selective attention and working memory in older adults: Results from a randomized controlled trial. Front Aging Neurosci. 2017;9:354 https://doi.org/10.3389/fnagi.2017.00354.

43. Powers K, Brooks P, Aldrich N, Palladino M, Alfieri L. Effects of video-game play on information processing: meta-analytic investigation. Psychon Bull Rev. 2013;20:1055-79 https://doi.org/10.3758/s13423-0418-z.

44. Lampit $\mathrm{A}$, Hallock $\mathrm{H}$, Valenzuela M. Computerized cognitive training in cognitively healthy older adults: a systematic review and meta-analysis of effect modifiers. PLoS Med. 2014;11(11):e1001756 https://doi.org/10.1371/ journal.pmed.1001756

45. Toril P, Reales JM, Ballesteros S. Video game training enhances cognition of older adults: a meta-analytic study. Psychol Aging. 2014;29:706-16 https:// doi.org/10.1037/a0037507.

46. Wang P, Han-Hui Liu HH, Zhu XT, Meng T, Hui-Jie L, Zuo XN. Action video game training for healthy adults: a meta-analytic study. Front Psychol. 2016; 7:907 https://doi.org/10.3389/fpsyg.2016.00907.

47. Vazquez FL, Otero P, Garcla-Casal JA, Blanco V, Torres AJ, Arrojo M. Efficacy of video game-based interventions for active aging. A systematic literature review and meta-analysis. PLoS One. 2018;13(12):e0208192 https:/doi.org/ 10.1371/journal.pone.0208192.

48. Sala G, Tatlidil S, Gobet F. Video game training does not enhance cognitive ability: a comprehensive meta-analytic investigation. Psych Bull. 2018;144(2): 111-39 https://doi.org/10.1037/bul0000139.

49. Barnes DE, Santos-Modesitt W, Poelke G, Kramer AF, Castro C, Middleton LE, Yaffe K. The Mental Activity and eXercise (MAX) trial: a randomized controlled trial to enhance cognitive function in older adults. JAMA Intern Med. 2013;173(9):797-804 https://doi.org/10.1001/jamainternmed.2013.189.

50. Linde K, Alfermann D. Single versus combined cognitive and physical activity effects on fluid cognitive abilities of healthy older adults: a 4-month randomized controlled trial with follow-up. J Aging Phys Act. 2014;22(3): 302-13 https://doi.org/10.1123/JAPA.2012-0149.

51. Barcelos N, Shah N, Cohen K, Hogan MJ, Mulkerrin E, Arciero PJ, et al. Aerobic and Cognitive Exercise (ACE) pilot study for older adults: executive function improves with cognitive challenge while exergaming. J Int Neuropsychol Soc. 2015;21(10):768-79 https://doi.org/10.1017/S1355617715001083.

52. Desjardins-Crepeau L, Berryman N, Fraser S, Vu TTM, Kergoat MJ, Li K, et al. Effects of combined physical and cognitive training on fitness and neuropsychological outcomes in healthy older adults. Clin Interv Aging. 2016;11:1287-99 https://doi.org/10.2147/CIA.S115711.

53. McDaniel MA, Bugg JM, Waldum ER, Dufault C, Meyer A, Binder EF, et al. Effects of cognitive training with and without aerobic exercise on cognitively demanding everyday activities. Psychol Aging. 2014;29(3):717-30 https://doi.org/10.1037/a0037363.

54. Ngandu T, Lehtisalo J, Solomon A, Levälahti E, Ahtiluoto S, Antikainen R, et al. A 2 year multidomain intervention of diet, exercise, cognitive training, and vascular risk monitoring versus control to prevent cognitive decline in at-risk elderly people (FINGER): a randomised controlled trial. Lancet. 2015; 385(9984):2255-63 https://doi.org/10.1016/S0140-6736(15)60461-5.

55. Lauenroth A, loannidis AE, Teichmann B. Influence of combined physical and cognitive training on cognition: a systematic review. BMC Geriatr. 2016; 16:141 https://doi.org/10.1186/s12877-016-0315-1.
56. Colcombe S, Kramer AF. Fitness effects on the cognitive function of older adults: a meta-analytic study. Psychol Sci. 2003;14(2):125-30 https://doi.org/ 10.1111/1467-9280.t01-1-01430.

57. Smith PJ, Blumenthal JA, Hoffman BM, et al. Aerobic exercise and neurocognitive performance: a meta-analytic review of randomized controlled trials. Psychosom Med. 2010;72(3):239-52 https://doi.org/10.1097/ PSY.0b013e3181d1e4633.

58. Voelcker-Rehage C, Niemann C. Structural and functional brain changes related to different types of physical activity across the life span. Neurosci Biobehav Rev. 2013;37(9):2268-95 https://doi.org/10.1016/j.neubiorev.2013. 01.028.

59. Hötting K, Rödder B. Beneficial effects of physical exercise on neuroplasticity and cognition. Neurosc Biobehav Rev. 2013;37:2247-57 https://doi.org/10. 1016/j.neubiorev.2013.04.005.

60. Niemann C, Godde B, Voelcker-Rehage C. Not only cardiovascular, but also coordinative exercise increases hippocampal volume in older adults. Front Aging Neurosc. 2014;6:170 https://doi.org/10.3389/fnagi.2014.0017.

61. Bamidis PD, Vivas AB, Styliadis C, et al. A review of physical and cognitive interventions in aging. Neurosc Biobehav Rev. 2014;44:206-20 https://doi. org/10.1016/j.neubiorev.2014.03.019.

62. Erickson Kl, Prakash RS, Voss MW, Chaddock L, Hu L, Morris KS, et al. Aerobic fitness is associated with hippocampal volume in elderly humans. Hippocampus. 2009;19(10):1030-9 https://doi.org/10.1002/hipo.20547.

63. Erickson KI, Voss MW, Prakash RS, Basak C, Szabo A, Chaddock L, et al. Exercise training increases size of hippocampus and improves memory. Proc Natl Acad Sci. U S A. 2011;108(7):3017-22 https://doi.org/10.1073/pnas. 1015950108.

64. Kattenstroth JC, Kolankowska I, Kalisch T, Dinse HR. Superior sensory, motor, and cognitive performance in elderly individuals with multi-year dancing activities. Front Aging Neurosci. 2010;2:31 https://doi.org/10.3389/fnagi.2010. 00031.

65. Kattenstroth JC, Kalisch T, Holt S, Tegenthoff M, Dinse HR. Six months of dance intervention enhances postural, sensorimotor, and cognitive performance in elderly without affecting cardio-respiratory functions. Front Aging Neurosci. 2013;5:5 https://doi.org/10.3389/fnagi.2013.00005.

66. Zilidou VI, Frantzidis CA, Romanopoulou ED, et al. Functional re-organization of cortical networks of senior citizens after a 24-week traditional dance program. Front Aging Neurosci. 2018;10:422 https://doi.org/10.3389/fnagi. 2018.00422.

67. Krampe RT, Smolders C, Doumas M. Leisure sports and postural control: can a black belt protect your balance from aging? Psychol Aging. 2014;29(1):95102 https://doi.org/10.1037/a0035501.

68. Muiños M, Ballesteros S. Peripheral vision and perceptual asymmetries in young and older martial arts athletes and nonathletes. Atten Percept Psychophys. 2014;76(8):2465-76 https://doi.org/10.3758/s13414014-0719-y.

69. Muiños M, Ballesteros S. Sport can protect dynamic visual acuity from aging: a study with young and older judo and karate martial arts athletes. Atten Percept Psychophys. 2015;77(6):2061-73 https://doi.org/10.3758/s13414-0150901-X.

70. Pons van Dijk G, Huijts M, Lodder J. Cognition improvement in Taekwondo novices over 40. Results from the SEKWONDO Study. Front Aging Neurosci. 2013;5:74 https://doi.org/10.3389/fnagi.2013.00074.

71. Wayne PM, Walsh JN, Taylor-Piliae RE, et al. Effect of Tai Chi on cognitive performance in older adults: systematic review and meta-analysis. J Am Geriatr Soc. 2014;62(1):25-39 https://doi.org/10.1111/jgs.12611.

72. Peter C, Kreisner A, Schröter M, Kim H, Bieber G, Öhberg F, Hoshi K, Waterworth EL, Waterworth J, Ballesteros, S. AGNES: Connecting people in a multidimensional way. J Multidimensional User Interfaces. https://doi.org/1 01007/s12193-0118-z. https://doi.org/10.1007/s12193-013-0118-z.

73. Ballesteros S, Toril P, Mayas J, Reales JM, Waterworth J. An ICT-mediated social network in support of successful ageing. Gerontechnology. 2014;13(1): 39-48 https://doi.org/10.4017/gt.2014.13.1.007.00.

74. Dause TJ, Kirby ED. Aging gracefully: social engagement joins exercise and enrichment as a key lifestyle factor in resistance to age-related cognitive decline. Neural Regen Res. 2019;14(1):39-42 https://doi.org/10.4103/16735374.243698.

75. Gajewski PD, Ferdinand NK, Kray J, Falkenstein M. Understanding sources of adult age differences in task switching: evidence from behavioral and ERP studies. Neurosci Biobehav Rev. 2018;92:255-75 https://doi.org/10.1016j. neubiorev.2018.05.029 
76. Gajewski PD, Freude G, Falkenstein M. Cognitive training sustainably improves executive functioning in middle-aged industry workers assessed by task switching: a randomized controlled ERP study. Front Hum Neurosci. 2017;11:81. https://doi.org/10.3389/fnhum.2017.00081.

77. Folstein ME, Folstein SE, McHugh PR. Mini-mental state. A practical method for grading the cognitive state of patients for the clinician. J Psychiatr Res. 1975;12(3):189-98.

78. Yesavage JA, Brink TL, Rose TL, Lum O, Huang V, Adey M, et al. Development a validation of a geriatric depression screening scale: a preliminary report. J Psychiatr Res. 1982-1983;17(1):37-49.

79. Martínez J, Onis MC, Dueñas H, Aguado C, Colomer C, Luque R. The Spanish version of the Yesavage Abbreviate Questionnaire (GDS) to screen depressive dysfunctions in patients older than 65. Medifam. 2002;12:620-30 https://doi.org/10.4321/S1131-57682002001000003.

80. Faul F, Erdfelder E, Buchner A, Lang A-G. Statistical power analyses using G*Power 3.1: tests for correlation and regression analyses. Behavior Res Methods. 2009;41:1149-60.

81. Falck RS, Davis JC, Best JR, Crockett RA. Impact of exercise training on physical and cognitive function among older adults. A systematic review and meta-analysis. Neurobiol Aging. 2019;79:119-30.

82. Montgomery AA, Peters TJ, Little P. Design, analysis and representation of factorial randomised controlled trials. BMC Med Res Meth. 2003;3:26. https:// doi.org/10.1186/1471-2288-3-26.

83. Chan S-W, Tetzaff JM, Gotzsche PC, et al. SPIRIT 2013 explanation and elaboration: guidance for protocols of clinical trials. BMJ Res Meth Report. 2013;346:e7586. https://doi.org/10.1136/bmj. e7586.

84. Boot WR, Simons DJ, Stothart C, Stutts $C$. The pervasive problem with placebos in psychology: why active control groups are not sufficient to rule out placebo effects. Persp Psycol Sci. 2013;8(4):24-254 https://doi.org/10. 1177/1745691613491271.

85. Saldanha IJ, Dickersin K, Wang X, Tianjing L. Outcomes in Cochrane systematic reviews addressing four common eye conditions: an evaluation of completeness and comparability. PLoS One. 2014;9(10):e109400. https:// doi.org/10.1371/journal.pone.0109400.

86. Zarin DA, Tse T, Williams RJ, et al. The status of trial registration eleven years after the ICMJE policy. NEJM. 2011;364:852-60.

87. Gajewski PD, Wild-Wall N, Schapkin SA, Erdmann U, Freude U, Falkenstein M. Effects of aging and job demands on cognitive flexibility assessed by task switching. Biol Psychol. 2010;85(2):187-99 https://doi.org/10.1016/j. biopsycho.2010.06.009.

88. Basak C, Boot WR, Voss MW, Kramer AF. Can training in real time strategy video game attenuate cognitive decline in older adults? Psychol Aging. 2008;23(4):765-77 https://doi.org/10.1037/a0013494.

89. Dahlin E, Nyberg L, Bäckman L, Stigsdotter Neely A. Plasticity of executive functioning in young and older adults: immediate gains, transfer, and longterm maintenance. Psychol Aging. 2008;23(4):720-30 https://doi.org/10. 1037/a0014296

90. Redondo MT, Beltrán-Brotóns JL, Reales JM, Ballesteros S. Executive functions in patients with Alzheimer's disease, type 2 diabetes patients and cognitively healthy older adults. Exp Gerontol. 2016;83:47-55 https://doi. org/10.1016/j.exger.2016.07.013.

91. Weschler Memory Scale (WMS-III) Copyright 1997 by Harcourt Assessment, Inc. San Antonio, TX, USA. Spanish Edition 2004 by TEA Ediciones, SA, Madrid (Spain).

92. Bell AJ, Sejnowski TJ. An information-maximization approach to blind separation and blind deconvolution. Neural Comput. 1995;7(6):1129-59.

93. Jung T, Makeig S, Mckeown MJ, Bell AJ, Lee T, Sejnowski TJ. Imaging brain dynamics using dependent component analysis. In: Proc IEEE Inst Electr Electron Eng. 2001 Jul 1 Presented at: Proceedings of the IEEE. Columbus; 2001. p. 1107-22 : http://europepmc.org/abstract/MED/20824156. https:// doi.org/10.1109/5.939827.

94. Makeig S, Bell A, Jung T, Sejnowski T. Independent component analysis of electroencephalographic data. Neural Netw. 2003;16(9):1311-23 https://doi. org/10.1016/j.neunet.2003.08.003.

95. Makeig S, Jung T, Bell AJ, Ghahremani D, Sejnowski TJ. Blind separation of auditory event-related brain responses into independent components. Proc Natl Acad Sci U S A. 1997;94(20):10979-84

96. Onton J, Delorme A, Makeig S. Frontal midline EEG dynamics during working memory. Neuroimage. 2005;27(2):341-56 https:/doi.org/10.1016/j.neuroimage.2005.

97. Lee T, Girolami M, Sejnowski TJ. Independent component analysis using an extended infomax algorithm for mixed subGaussian and superGaussian sources. Neural Comput. 1999;11(2):417-41.
98. Jung TP, Makeig S, Stensmo M, Sejnowski TJ. Estimating alertness from the EEG power spectrum. IEEE Trans Biomed Eng. 1997;44(1):60-9 https://doi. org/10.1109/10.553713.

99. Watson D, Clark LA, Tellegen A. Development and validation of brief measures of positive and negative affect: The PANAS Scales. J Pers Soc Psychol. 1988;54(6):1063-70 PMID: 3397865.

100. Sandín B, Chorot P, Lostao L, Joiner TE, Santed ME, Valiente RM. Escalas PANAS de afecto positivo y negativo: validación factorial y convergencia transcultural. Psicothema. 1999:11:37-51.

101. Nolla MC, Queral R, Miró J. Las escalas PANAS de afecto positivo y negativo: nuevos datos de su uso en personas mayores. Revista de Psicopatología y Psicología Clínica. 2014;19(1):15-21.

102. Neugarten BL, Havighurst RJ, Tobin SS. The measurement of life satisfaction. J Gerontol. 1961;16:134-43 PMID: 13728508.

103. Guralnik JM, Simonsick EM, Ferrucci L, Glynn RJ, Berkman LF, Blazer DG, et al. A Short Physical Performance Battery assessing lower-extremity function-association with self-reported disability and prediction of mortality and nursing-home admission. J Gerontol. 1994;49(2):M85-94 PMID: 8126356.

104. Harada ND, Chiu V, Stewart AL. Mobility-related function in older adults: assessment with a 6-Minute Walk Test. Arch Phys Med Rehabil. 1999:80(7): 837-41 PMID: 10414771

105. ATS Committee on proficiency standards for clinical pulmonary function laboratories. ATS statement: guidelines for the Six-Minute Walk Test. Am J Respir Crit Care Med. 2002;166(1):111-7 https://doi.org/10.1164/ajrccm.166.1. at1102.

106. Borg GA. Psychophysical bases of perceived exertion. Med Sci Sports Exerc. 1982;14(5):377-81 PMID: 7154893.

\section{Publisher's Note}

Springer Nature remains neutral with regard to jurisdictional claims in published maps and institutional affiliations.

Ready to submit your research? Choose BMC and benefit from:

- fast, convenient online submission

- thorough peer review by experienced researchers in your field

- rapid publication on acceptance

- support for research data, including large and complex data types

- gold Open Access which fosters wider collaboration and increased citations

- maximum visibility for your research: over $100 \mathrm{M}$ website views per year

At BMC, research is always in progress.

Learn more biomedcentral.com/submissions 